\title{
Library of Visual Descriptors and Machine Learning Algorithms for Texture and Text Detection
}

\author{
Jhonatas S. Conceição*, Allan Pinto, and Ricardo da S. Torres
}

\begin{abstract}
In this article, we present the implementation of a solution for the text localization problem. More precisely, the solution presented in this article implements a prototype containing approaches for image characterization based on color and texture visual properties for supporting text region classification. As a result, we present a comparison study of several classifications systems for detecting text regions.
\end{abstract}

\section{Key words:}

Text Localization, Image classification, Scene Text Detection

\section{Introduction}

Text detection and recognition is one of many problems in computer vision which consists of identifying characters and words in images or videos. This is a important topic in computer vision since this text localization and recognition can benefit several moderns applications such as traffic sign, assistive computing, among others. This article presents a method for text detection using different methods for image characterization such as Local Binary Pattern, Gabor Filter, Histogram of Oriented Gradients and Border/Interior Pixel Classification combined with different classifiers such as Support Vector Machine and Random Forest. We implement a library containing a set of methods for image characterization, based on color and texture visual properties, in order to create a classification system for detecting text regions in images.

\section{Results and Discussion}

This article presents a prototype related to the implementation of techniques for detecting textual elements based on texture cues. This prototype contains the implementation of four texture- and colorbased visual representation methods and two classification algorithms. In this section, we briefly describe these methods: (a) Local Binary Pattern (LBP): This feature descriptor encodes textural information in binary codes [1]; (b) Gabor Filter (GF): This feature descriptor comprises of several band-pass filters, whose impulse response is generated by multiplying a Gaussian function with an oscillating function such as sinusoidal waves [2]; (c) Histogram of Oriented Gradient (HOG): This feature descriptor encodes local features by counting occurrences of gradient orientation in localized regions of an image, which can be defined using sliding windows or points of interest [2]; and (d) Border/Interior Pixel Classification (BIC): This feature descriptor encodes color information by using color histograms considering two classes of pixels: border and interior [3].

The feature descriptor methods were evaluated along with Support Vector Machine (SVM) and Random Forest (RF) algorithms, as illustrated in Table 1. As we could observe, the BIC descriptor presented the best results in terms of precision, recall, and f-measure for both SVM and RF algorithms. Different from the other visual descriptors, the BIC encode textural and color information of images in two separate histograms to distinguish border information from interior (non-border) regions of images. Also, the BIC descriptor produces a very compact representation, which makes this method a good choice for a restrictive scenario computing.

Table 1. Comparison results among different methods for image characterization and text region classification.

\begin{tabular}{|l|l|c|c|c|c|c|c|c|}
\hline & $\begin{array}{l}\text { LBP+ } \\
\text { SVM } \\
(\%)\end{array}$ & $\begin{array}{c}\text { LBP+ } \\
\text { RF } \\
(\%)\end{array}$ & $\begin{array}{c}\text { HOG+ } \\
\text { SVM } \\
(\%)\end{array}$ & $\begin{array}{c}\text { HOG+ } \\
\text { RF } \\
(\%)\end{array}$ & $\begin{array}{c}\text { BIC+ } \\
\text { SVM } \\
(\%)\end{array}$ & $\begin{array}{c}\text { BIC+ } \\
\text { RF } \\
(\%)\end{array}$ & $\begin{array}{c}\text { GF+ } \\
\text { SVM } \\
(\%)\end{array}$ & $\begin{array}{c}\text { GF+ } \\
\text { RF } \\
(\%)\end{array}$ \\
\hline P & 70.34 & 80.48 & 85.88 & 86.28 & 99.58 & 99.82 & 77.13 & 81.98 \\
\hline R & 69.78 & 68.05 & 83.24 & 76.82 & 99.58 & 99.65 & 75.87 & 63.93 \\
\hline F & 69.57 & 64.42 & 83.70 & 77.72 & 99.58 & 99.82 & 76.23 & 63.08 \\
\hline
\end{tabular}

\section{Conclusions}

This article describes experiments designed to evaluate the proposed solutions for text detection. As a result, a library of classifiers with emphasis on character detection was created. The best results achieved in this work were obtained by combining BIC descriptor with Random Forest classifier, which reaches a precision, recall and $\mathrm{f}$ measure of $99.82 \%, 99.65 \%$, and $99.82 \%$, respectively, considering the ICDAR2003 dataset.

\section{Acknowledgment}

This article is related to the ongoing project named MultiLingual Text Spotting and Recognition (MLTSR), which is being developed in the context of the collaboration between Samsung R\&D Institute Brazil (SRBR) and University of Campinas (Unicamp).

1T. Ojala, M. Pietikainen, and T. Maenpaa, "Multiresolution gray-scale and rotation invariant texture classification with local binary patterns,” IEEE Trans. on Pattern Anal. Mach. Intell., vol. 24, no. 7, pp. 971-987, July 2002.

${ }^{2} \mathrm{X}$. Wang, X. Ding, and C. Liu, "Gabor filters-based feature extraction for character recognition,” Pattern Recogn., vol. 38, no. 3, pp.369-379, Mar. 2005.

${ }^{3}$ R. O. Stehling, M. A. Nascimento, and A. X. Falcão, “A compact and efficient image retrieval approach based on border/interior pixel classification,” in ACM 11th Intl. Conf. on Information and Knowledge Management, 2002, pp. 102-109. 